\title{
Burden of Cryptosporidium Infections in the Yangtze River Delta in China in the 21st Century: A One Health Perspective
}

\author{
Jianhai Yin, Yujuan Shen and Jianping Cao*
}

\begin{abstract}
Cryptosporidiosis is a leading cause of diarrheal disease in some populations, including young children and people with compromised immune systems. The epidemiology of Cryptosporidium, which is transmitted mainly through waterborne routes, has been a serious public health concern. Cryptosporidiosis is closely associated with animals and the shared environment, and is well suited to a One Health approach to prevention and control. In China, Cryptosporidium investigations in humans, various animal species, water bodies and other environments have been widely conducted, including in the Yangtze River Delta, which encompasses Shanghai, Jiangsu, Zhejiang and Anhui. With the increasing integrated development of the Yangtze River Delta, advance preparation and effective monitoring are necessary to prevent outbreaks of neglected tropical diseases, such as cryptosporidiosis, and to contribute to infectious disease prevention and control in the entire region. Moreover, the epidemiological surveillance of infectious diseases is a critical public health measure. This article reviews the burden of Cryptosporidium in the Yangtze River Delta at the human-animal-environment interface, as reported since 2001, and identifies the deficiencies and challenges in epidemiological studies of Cryptosporidium in this region from a One Health perspective, to provide basic information for the formulation of prevention and control strategies.
\end{abstract}

Keywords: Cryptosporidium, zoonotic, epidemiology, Yangtze River Delta, One Health

\section{BACKGROUND}

Cryptosporidium is a genus of protozoan parasites infecting a wide host range including humans, domesticated livestock and poultry, companion animals and wildlife [1]. More than 40 species of Cryptosporidium have been confirmed; of these, 19 species (Cryptosporidium hominis, C. parvum, C. meleagridis, C. canis, C. felis, C. ubiquitum, C. cuniculus, C. viatorum, C. muris, C. andersoni, C. suis, C. erinacei, C. bovis, C. tyzzeri, C. occultus, C. ditrichi, C. fayeri, C. xiaoi and C. scrofarum) and four genotypes (chipmunk genotype I, horse genotype, skunk genotype and mink genotype) have been found to be zoonotic $[2,3]$. The first five species are the most frequently detected species in humans $[1,3,4]$. Cryptosporidium is transmitted mainly through ingestion of oocysts in fecally contaminated water or food, or through direct contact with infected animals or people. Oocysts are excreted by the infected host mainly through the feces. The sporozoites are then released, then
Edited by:

Su Han, Harbin Medical University: Both reviewers chose to be anonymous.

*Corresponding author: E-mail: caojp@yahoo.com (JC)

National Institute of Parasitic Diseases, Chinese Center for Disease Control and Prevention (Chinese Center for Tropical Diseases Research); Key Laboratory of Parasite and Vector Biology, National Health Commission of the People's Republic of China; World Health Organization Collaborating Centre for Tropical Diseases; National Center for International Research on Tropical Diseases, Shanghai 200025, People's Republic of China

Received: December 42021 Revised: January 172022 Accepted: January 252022 Published Online: February 172022 
parasitize primarily gastrointestinal epithelial cells and possibly also respiratory epithelial cells. Oocysts develop and sporulate through asexual multiplication and sexual multiplication, and thick-walled oocysts are then excreted from the host into the environment. However, the infection and disease caused by this parasite remain neglected worldwide [5], although cryptosporidiosis is a leading cause of diarrheal disease in some populations, including young children and people with compromised immune systems [6,7]. Unfortunately, no vaccines are available to prevent this infection or disease in humans or in livestock, and effective treatment measures are lacking. Moreover, the only approved drug, nitazoxanide, has an elevated failure rate in malnourished children [7,8]. In addition, Cryptosporidium spp. have been found to be common causes of waterborne outbreaks of diarrheal illness globally, after the contamination of drinking and recreational water with the oocyst stage from host feces [9-11]. Therefore, cryptosporidiosis is closely associated with animals and the shared environment, and is well suited to a One Health approach with interdisciplinary collaboration to effectively manage this disease [1214]. This strategy has been demonstrated to address health issues, including zoonotic diseases, at the human-animal-environment interface, [15].

In China, Cryptosporidium investigations in humans, various animal species, water bodies, and other environments have been widely conducted, including in the Yangtze River Delta, a world-class city cluster in China. It encompasses the metropolis of Shanghai and the provinces of Jiangsu, Zhejiang and Anhui, and is one of the most economically developed and populated regions in China (https://govt. chinadaily.com.cn/index/specials/theyangtzeriverdelta). Recently, an increasingly integrated health service system with a unified public network for infectious disease prevention and control has been established, according to an agreement signed at the first Yangtze River Delta Health Summit (https://www.shine.cn/news/metro/1905114499/). This agreement will strengthen inter-provincial cooperation in health care, including serious public health case management, cross-provincial epidemiological investigation and information sharing. The surveillance of infectious intestinal diseases is a critical public health measure that can be used to understand the epidemiology of diarrheal disease. Reports have described human and animal infections with Cryptosporidium spp., and the contamination of various water bodies with Cryptosporidium spp. in this region. These reports have additionally demonstrated how measures to address neglected tropical diseases such as cryptosporidiosis can be planned in advance, and how monitoring can effectively prevent outbreaks.

Here, we systematically review the burden of Cryptosporidium spp. in the Yangtze River Delta at the humananimal-environment (water) interface, as reported since 2001 in the PubMed and Chinese databases (Wanfang, CNKI,VIP). We identify the deficiencies and challenges in epidemiological studies on Cryptosporidium in this region from a One Health perspective.

\section{CRYPTOSPORIDIUM INFECTIONS IN HUMANS}

People are generally susceptible to Cryptosporidium, and experience watery diarrhea caused by tiny, one-celled Cryptosporidium oocysts that enter the body, travel primarily to the gastrointestinal tract and are finally excreted through the feces. A clear understanding of the epidemiology of Cryptosporidium is an important prerequisite for preventing potential outbreaks. In China, an average prevalence of $2.97 \%$ involving at least 200,054 people from 27 provinces has been revealed in a retrospective epidemiological analysis of human Cryptosporidium infections (1987-2018); the burden of disease caused by Cryptosporidium varies between and within areas [16]. In the Yangtze River Delta, where human cryptosporidiosis in China was first reported in 1987 [17], many investigations of Cryptosporidium in humans have been conducted in the $21^{\text {st }}$ century (Fig 1).

A variety of populations including people with AIDS (Huaibei) [18], HIV-positive individuals and HIV-negative individuals in HIV/AIDS high-endemic areas (Fuyang) [19-22], students in various age groups (Hefei, Bengbu, Huainan, Lu'an, Wuhu, Huaibei, Huangshan, Fuyang, Chuzhou, Anqing and Suzhou) [23,24], infants (Huainan) [25], patients with diarrhea (Huainan) [26] and the general population (Anqing and Suzhou) [27] in Anhui have been investigated for the detection of Cryptosporidium oocysts through traditional methods, such as modified acidfast staining (5), phenol-auramine staining (2), phenolauramine plus modified acid-fast staining (3), direct fecal smears (1) and enzyme-linked immunosorbent assays (1). Infection rates have been identified as follows: patients with AIDS (1/139), HIV-positive individuals (21/302-8/79), HIV-negative individuals $(9 / 294,9 / 303)$, people in HIV/ AIDS high-endemic areas (32/720), kindergarten children $(21 / 889$ or $28 / 889,30 / 1,024)$, primary school students $(7 / 1,098$ or $9 / 1,098)$, middle school students $(7 / 1,092$ or $9 / 1,092)$, college students $(5 / 969$ or $8 / 969)$, infants $(7 / 203)$, patients with diarrhea $(46 / 827)$ and the general population (6/331). However, no data are available on the Cryptosporidium species, genotypes and subtypes infecting humans in Anhui province.

In Jiangsu, patients with diarrhea (Xuzhou, Zhenjiang) [28,29], children in outpatientsettings (Nanjing) [30] and rural areas (unreported) [31,32], residents (Nanjing, Changzhou) $[33,34]$ and intravenous drug users (Nanjing) [35] have been selected for the investigation of Cryptosporidium infection status through modified acid-fast staining (2), phenolauramine staining (1), phenol-auramine plus modified acidfast staining (5), nested polymerase chain reaction based on the Cryptosporidium 18S rRNA gene (subsequently referred to as nested PCR) (3) and indirect enzyme-linked immunosorbent assays (1). The reported infection rates of the various groups are as follows: patients with diarrhea (0/96-23/232), children $(22 / 2,268-18 / 1,648)$, residents $(0 / 581,18 / 1637)$ and intravenous drug users (411/588). The zoonotic species of C. andersoni and C. hominis have been identified in patients with diarrhea in Zhenjiang, and the former species 


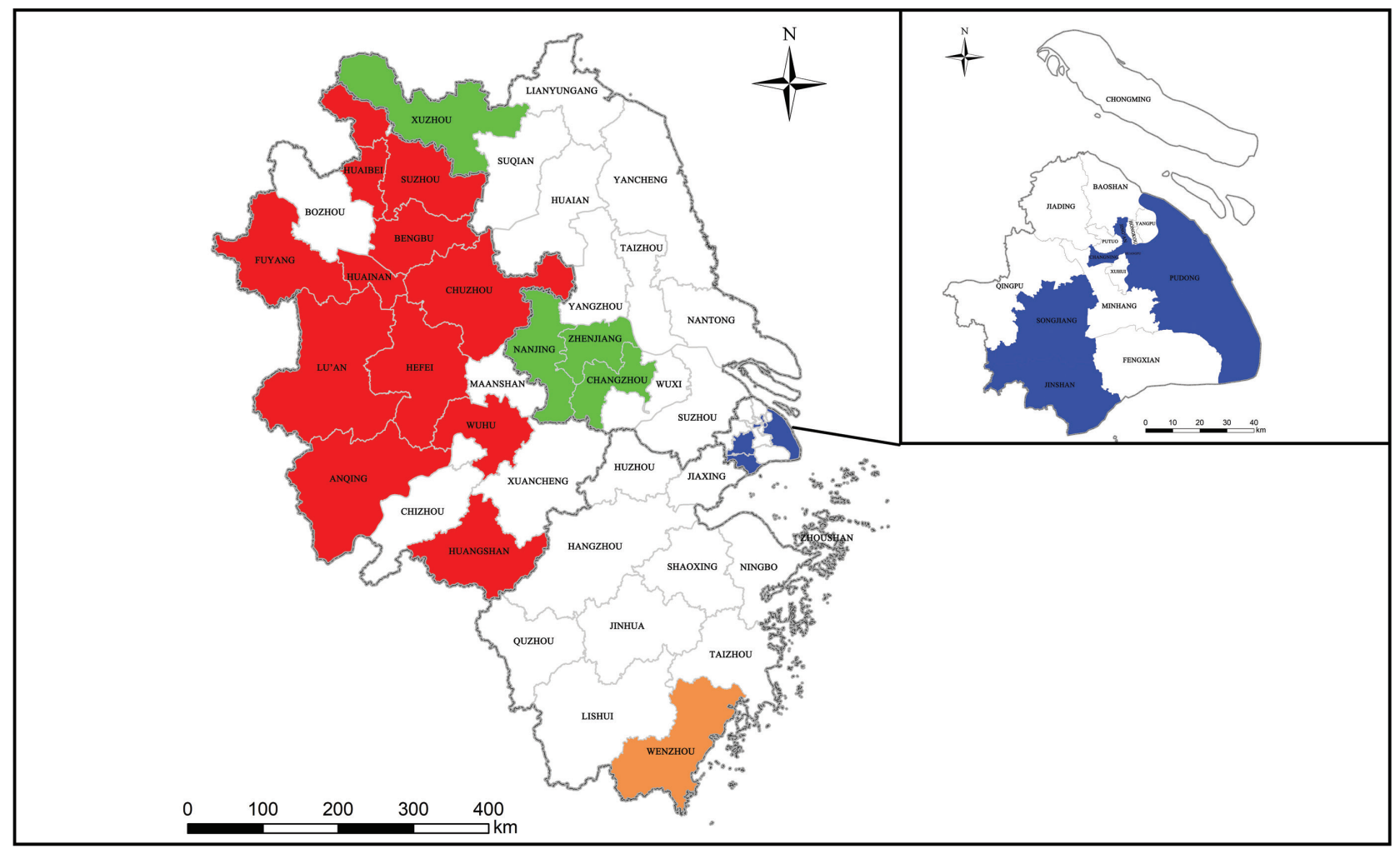

Figure 1 | Cryptosporidium investigations in humans at the prefectural level in the Yangtze River Delta since 2001. Red, green, blue and orange represent the prefecture-level cities/districts in Anhui, Jiangsu, Shanghai and Zhejiang, respectively, where Cryptosporidium surveys in humans were performed.

is considered a novel predominant Cryptosporidium species in outpatients with diarrhea [29].

In Shanghai, Cryptosporidium infections have been investigated in patients with diarrhea in Luwan (now Huangpu) [36], Hongkou, Minhang, Changning, Jinshan $[37,38]$ and Pudong [39], and in general residents and residents who keep dogs in Luwan [36]. The main methods used were modified acid-fast staining (3), indirect immunofluorescence assays (1) and nested PCR (1). The reported infection rates ranged from $1.31 \%$ to $13.49 \%$ in patients with diarrhea and $13.33 \%$ in residents who keep dogs, and no infections were found in the general residents. The infections in patients with diarrhea in Pudong were determined to be caused by C. andersoni [39]. In addition, a total of 102 of 6,284 patients in three pediatric hospitals in Shanghai were reported to be infected with Cryptosporidium between September 2007 and October 2009, on the basis of PCR and restriction fragment length polymorphism analysis of the small subunit rRNA gene. A total of 92 of 102 cases were infected with C. hominis; six cases were infected with C. meleagridis; and C. canis and C. felis were found in two cases each [40]. Furthermore, a total of six C. hominis subtypes were identified (IaA14R4, IdA19, IbA19G2, IdA14, IaA18R4 and IgA14) through sequence analysis of the 60-kDa glycoprotein gene.

In Zhejiang, one case of intracranial infection of Cryptosporidium was reported in Wenzhou in 2003 [41]; however, no further reports have been made in the $21 \mathrm{st}$ century.
In brief, the zoonotic species of C. andersoni, C. hominis, C. meleagridis, C. canis and C. felis, and six C. hominis subtypes (IaA14R4, IdA19, IbA19G2, IdA14, IaA18R4 and IgA14) have been identified in humans in this region.

\section{CRYPTOSPORIDIUM INFECTIONS IN ANIMALS}

Livestock and wildlife have been demonstrated to be relevant contributors to zoonotic Cryptosporidium oocysts in environments in which they are intended for human consumption. Cryptosporidiosis is a major diarrheal disease in neonatal calves and other animals.

\section{Cattle}

Cattle are an important source of zoonotic cryptosporidiosis, and cryptosporidiosis frequently occurs in cattle in China $[42,43]$. Several investigations of Cryptosporidium infections in cattle have been conducted in the Yangtze River Delta (Fig 2).

In Anhui, C. muris and C. andersoni have been found in three epidemiological surveys of cryptosporidiosis in dairy cattle, with infection rates ranging from 5/285 to $103 / 325$ in Hefei through modified acid-fast staining, the saturated saline floatation method and nested PCR [44-46]. In another survey, 26 Cryptosporidium-positive fecal samples ( $C$. muris and C. parvum) have been identified among 502 dairy cattle [47]. In addition, C. muris and C. parvum have been detected in cattle from Bozhou (20/144), Suzhou (22/101) 


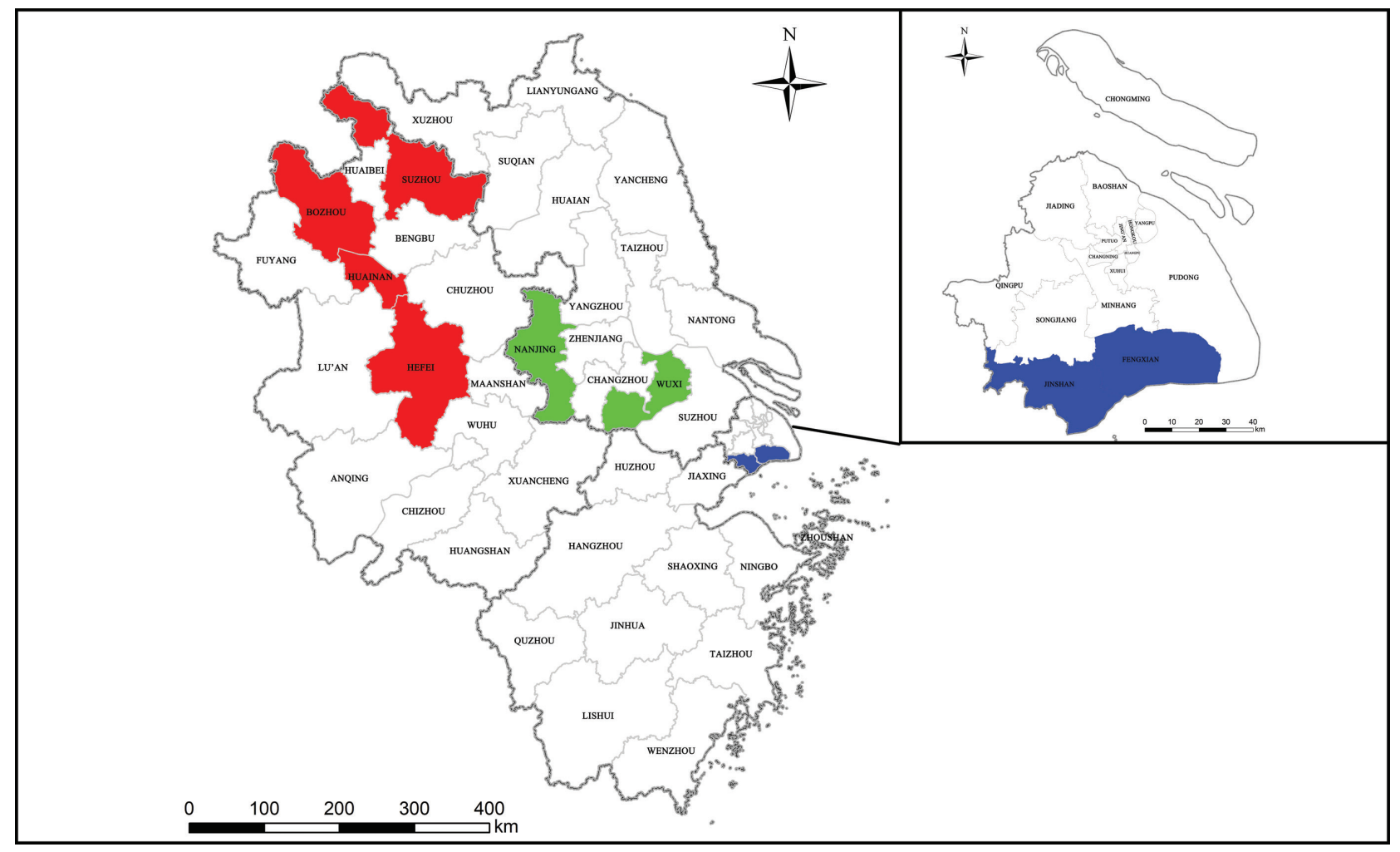

Figure 2 | Cryptosporidium investigations in cattle at the prefectural level in the Yangtze River Delta since 2001. Red, green and blue represent the prefecture-level cities/districts in Anhui, Jiangsu and Shanghai, respectively, where the Cryptosporidium surveys in cattle were performed.

and Huainan $(0 / 30)$ with the saturated saline floatation method [48].

In Jiangsu, Cryptosporidium infection rates of 34/191 [49], 9/279 [50] and 44/130 [51] have been found in dairy cattle from Nanjing in three reports, through detection with modified acid-fast staining, the saturated saline floatation method and nested PCR. C. bovis, C. ryanae and C. parvum have been confirmed by PCR in one study [51]. A total of 27 Cryptosporidium-positive samples have been identified in 126 dairy cattle fecal samples in Wuxi with the saturated saline floating method [52]. In another study, 195 positive samples (C. parvum, C. ryanae, C. bovis and C. andersoni) from 1,372 neonatal calves on a dairy farm have been characterized with nested PCR [53].

In Shanghai, a Cryptosporidium infection rate of 214/586 in dairy cattle has been reported in a survey using the saturated sucrose solution floating method and modified acid-fast staining [54]. Moreover, C. bovis, C. ryanae and C. parvum have been identified in longitudinal monitoring of Cryptosporidium species in pre-weaned dairy calves on five farms (303/818) with nested PCR, and further subtype analysis has identified C. parvum IIdA19G1 [55].

One joint investigation of Cryptosporidium in dairy cattle has been conducted in Anhui, Jiangsu and Shanghai with the modified acid-fast staining method and nested PCR.The reported infection rates were 69/350, 251/1315 and 67/491. The identified species were C. parvum "mouse" genotype, $C$. andersoni, C. bovis, C. hominis and C. serpentis [56].
Therefore, four zoonotic species (C. muris, C. andersoni, C. parvum and C. bovis) and other species (C. ryanae and C. serpentis), C. parvum "mouse" genotype and C. parvum IIdA19G1 have been characterized in cattle in this region.

\section{Sheep/goats}

Sheep and goats are intermediate hosts of Cryptosporidium, and the Cryptosporidium infection of sheep and goat flocks in China is general [57]. Sheep are regarded as a potential source of zoonotic cryptosporidiosis in China [58]. Several surveys on Cryptosporidium infections in sheep and goats have been performed in this region (Fig 3).

In Anhui, only sheep samples from Bengbu (11/345) and Huainan $(10 / 52)$ have been observed to be positive for Cryptosporidium in the investigations conducted in Lu'an, Fuyang, Bengbu, Anqing, Maanshan and Huainan. C. xiaoi has been characterized in Bengbu and C. xiaoi and C. parvum in Huainan, and subtype analysis has identified C. parvum IIaA15G2R1 [58,59]. No positive Cryptosporidium samples have been identified in 80 goat fecal samples from Hefei with the saturated sucrose solution floatation method [50], whereas Cryptosporidium positive samples have been found in goat feces from $\mathrm{Si}$ County in Suzhou (18/50) and Wuhu (1/48) through modified acid-fast staining in surveys in Suzhou, Lu'an, Bozhou, Fuyang, Chizhou, Wuhu and Chuzhou [60]. C. parvum (IIdA19G1) has been identified in samples from Fuyang, Lu'an and Maanshan [59]. 


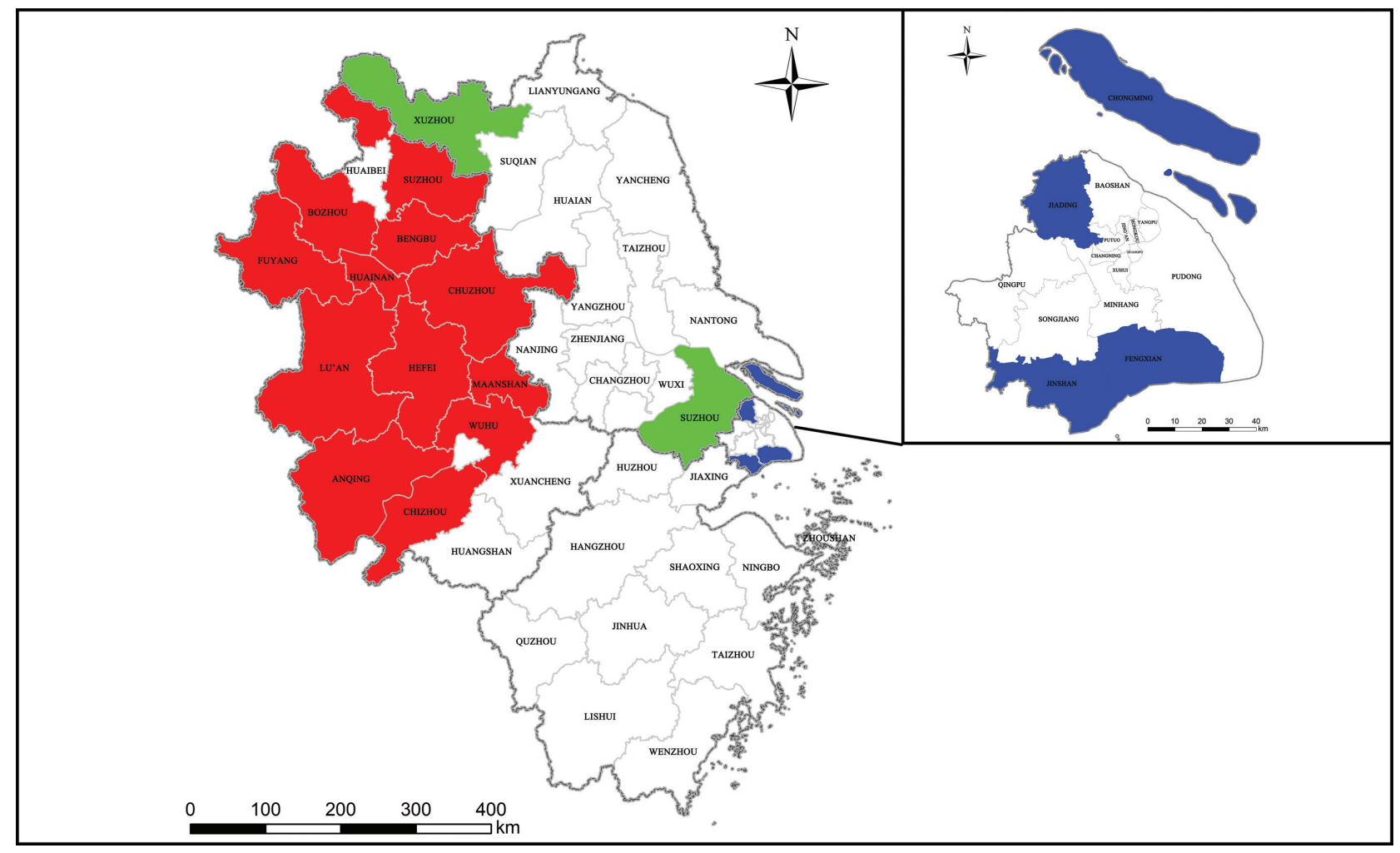

Figure 3 | Cryptosporidium investigations in sheep and goats at the prefectural level in the Yangtze River Delta since 2001. Red, green and blue represent the prefecture-level cities/districts in Anhui, Jiangsu and Shanghai, respectively, where Cryptosporidium surveys in sheep and goats were performed.

In Jiangsu, nested PCR has been used to detect Cryptosporidium in goats in Xuzhou $(0 / 74)$ and sheep in Suzhou (37/75). The positive samples have been found to be infected with C.xiaoi and C. ubiquitum; further subtype analysis of the latter has identified C. ubiquitum XIIa [59].

In Shanghai, nested PCR has also been used to test Cryptosporidium in goats (33/302) in Chongming, Jinshan and Fengxian [61], and in sheep (86/294) from Jiading [58], which have been found to be infected with C. xiaoi, C. parvum and C. ubiquitum. Furthermore, C. parvum IIdA19G1 and C. ubiquitum XIIa have been identified in Jiading through sequence analysis of the $60-\mathrm{kDa}$ glycoprotein gene [58].

Only one of these surveys was performed jointly between Huainan (Anhui) and Jiading (Shanghai) [58].

In summary, C. parvum (including IIdA19G1), C. xiaoi and C. ubiquitum (including XIIa) have been identified in sheep and goats in this region.

\section{Pigs}

Many studies on Cryptosporidium infection in pigs have been reported worldwide, and cryptosporidiosis is widespread in pigs in China [62]. Cryptosporidium infections in pigs in the Yangtze River Delta have also been investigated (Fig 4).

In Anhui, two wide-coverage investigations of Cryptosporidium infections in pigs have been conducted with the saturated sucrose solution floating method (Suzhou, Fuyang, Bozhou, Anqing, Chuzhou, Huangshan, Hefei,
Lu'an, Chizhou and Xuancheng) [63] and nested PCR (plus Huainan, but not in Suzhou, Chizhou, Xuancheng and Huangshan) [64]. An infection rate ranging from $6.42 \%$ to $26.19 \%$ has been detected with the former method in the same areas except Lu'an, Dongzhi County (Chizhou) and Xuanzhou (Xuancheng). Positive samples have been found only in Qianshan County (Anqing) (20/50) and Langya (Chuzhou) (4/64) with PCR, all C. scrofarum [64]. In addition, 92 of 162 samples have been found to be Cryptosporidium-positive, according to the auramine-phenol modified acid-fast staining method in another survey in the Fengtai County of Huainan [65].

In Jiangsu, modified acid-fast staining and reverse-transcription PCR have been used to investigate Cryptosporidium infection in pigs from Nanjing, Huai'an, Suqian and Wuxi; the infection rate has been reported to range from $11.02 \%$ to $14.17 \%$. C. parvum has been reported on the basis of the morphology of oocysts [66].

In Shanghai, the saturated sucrose solution floating method (312/1510) [67], modified acid-fast staining combined with nested PCR (14.29\%-90.57\%) [68] and modified acid-fast staining combined with reverse transcription PCR (28/273 in Qingpu and 30/267 in Jinshan) [66] have been used to investigate Cryptosporidium infection in pigs in Jinshan, Minhang, Songjiang, Qingpu, Jiading, Fengxian, Nanhui, Pudong, Chongming and Baoshan. C. suis and Cryptosporidium pig genotype II (recently C. scrofarum) have been found in Fengxian, Jiading, Minhang, 


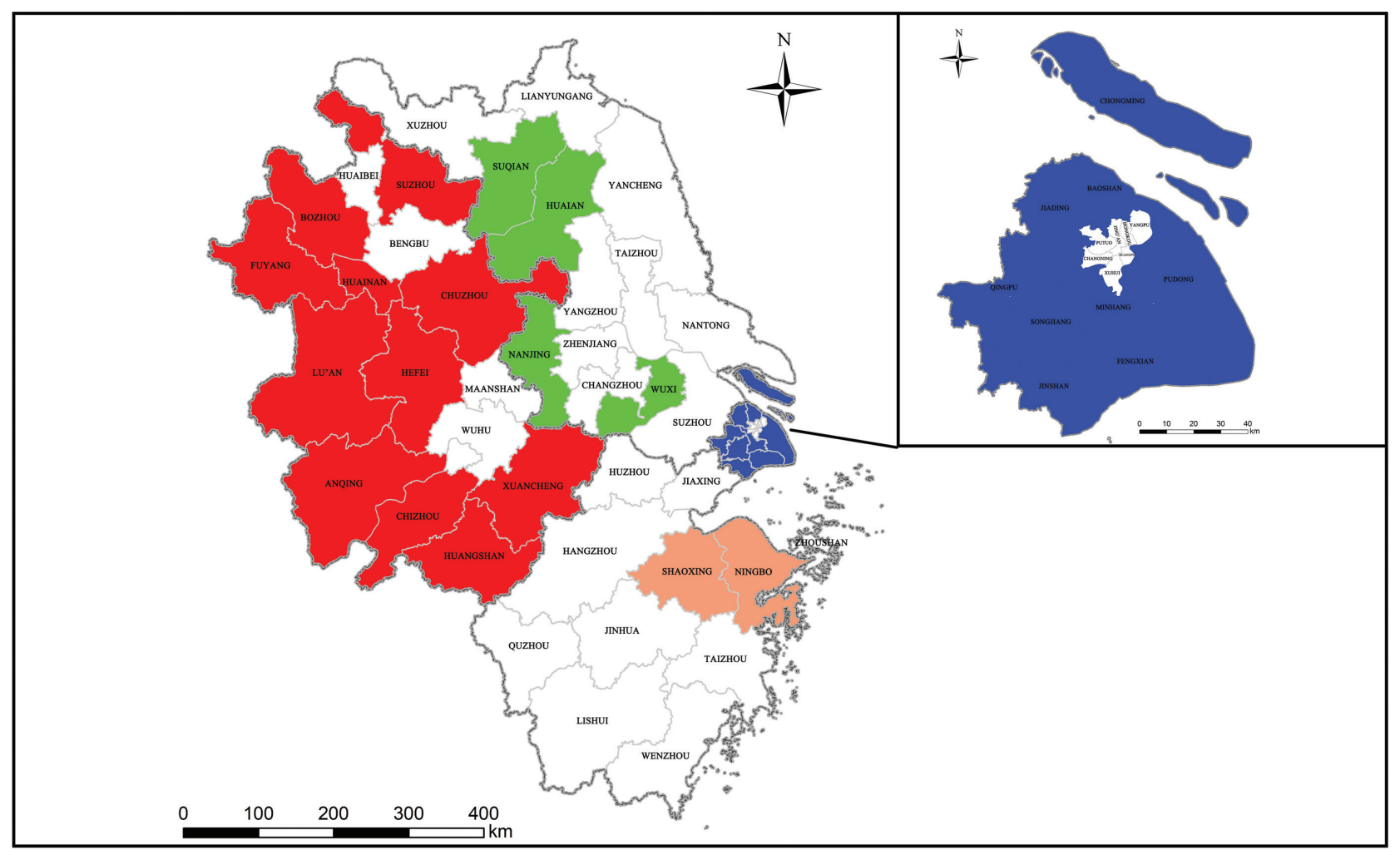

Figure 4 | Cryptosporidium investigations in pigs at the prefectural level in the Yangtze River Delta since 2001. Red, green, blue and orange represent the prefecture-level cities/districts in Anhui, Jiangsu, Shanghai and Zhejiang, respectively, where Cryptosporidium surveys in pigs were performed.

Pudong and Qingpu, but only C. suis has been found in Baoshan, Chongming and Songiiang [68]. Another species of C. parvum has been identified in Jinshan and Qingpu [66]. Additionally, two other reports have indicated infection with C. suis and/or Cryptosporidium pig genotype II [69,70].

In Zhejiang, nested PCR has been used to investigate Cryptosporidium infections in pigs from Shaoxing and Ningbo, with infection rates ranging from $0.93 \%$ to $29.10 \%$ [69-71]. C. suis and Cryptosporidium pig genotype II have been found in Shaoxing [69,70], and C. parvum has been identified in pigs slaughtered in Ningbo and transported from north China [71].

One of the above surveys was performed in both Jiangsu and Shanghai [66], and two were jointly conducted in Shanghai and Shaoxing (Zhejiang) [69,70].

Briefly, C. scrofarum (also Cryptosporidium pig genotype II), C. suis and C. parvum have been characterized in pigs in this region.

\section{Animals in zoos and shelters and pets}

In Anhui, the saturated sucrose solution floating method and modified acid-fast staining have been applied to detect Cryptosporidium infections in primates $(6 / 44,1 / 44)$ and herbivores $(2 / 41,3 / 43)$ in a zoo in Anhui. In this survey, primates were observed to be infected with C. hominis, and herbivores were found to be infected with C. andersoni [72]. The saturated sucrose solution floating method, modified acid-fast staining and nested PCR have been used jointly in Hefei Wildlife Park $(5 / 38,4 / 24)[73,74]$ and in a zoo in Anhui (6/44, C. hominis) [75]. C. muris and C. parvum have been identified in dogs in two surveys in Hefei with the saturated sucrose solution floating method (24/232) [76] and auramine-phenol modified acid fast staining (20/69) [77]. No positive samples $(0 / 215)$ have been identified in pet dogs from five prefectures in Anhui with nested PCR [78]. In addition, nested PCR has been used to detect Cryptosporidium in domestic cats in Lu'an (0/9) and Hefei (2/60), and C. felis has been detected in Hefei [79].

In Jiangsu, a total of 20 Cryptosporidium-positive samples have been found in 105 samples of dog feces in Nanjing with modified acid-fast staining [49]. Cat fecal samples from Nanjing (0/39) and Yangzhou (4/98) have been detected by nested PCR, and C. felis has been found in Yangzhou [79].

In Shanghai, an investigation of Cryptosporidium infections in reptiles and amphibians (wild snakes, lizards, turtles, giant tortoises and pythons) in the Shanghai Zoo and wildlife rescue stations with modified acid-fast staining has revealed a Cryptosporidium infection rate of 20/46 [80]. C. andersoni in non-human primates (3/83) [81] and C. canis in dogs and other animals (6/84) [82] have been identified in the Shanghai Zoo with phenol-auramine plus modified acid-fast staining and nested PCR, but no positive results have been found in 130 samples of various animal feces from Shanghai Wildlife Park [83]. Different infection rates with C. canis and C. parvum have been detected with nested PCR in dogs in canine shelters (Xuhui, Pudong, Qingpu, 
Minhang, Baoshan and Putuo) (8/442) [84] and in domestic pet dogs (Xuhui, Minhang and Putuo) (3/338-39/485) $[85,86]$. In addition, no positive cat fecal samples collected in Minhang (0/14) have been identified [79], whereas two positive samples (C. muris, C. hominis) have been identified in 290 monkey stool samples with nested PCR [87].

In Zhejiang, C. canis (5/100) and C.felis (2/81) have been identified in pet dog and cat fecal samples with nested PCR in Hangzhou [78,79], and three samples have been found to be positive (Cryptosporidium galli-like 1, Cryptosporidium galli-like 2 and Cryptosporidium avian genotype V-like) among 52 fecal samples from various animals in a zoo [83].

\section{Other animals}

In Anhui, 35 of 180 broiler chicken fecal samples from Huainan have been observed to be positive for Cryptosporidium with phenol-auramine plus modified acidfast staining [88] and 2 of 491 fecal samples from Chinese alligators (Alligator sinensis) from Xuancheng have been determined to be positive for C. testudinis with nested PCR [89].

In Jiangsu, 22, 17 and 13 samples have been reported to be positive for Cryptosporidium in the fecal samples of 156 rabbits, 102 geese and 168 chickens, respectively, with modified acid-fast staining in the investigation of Cryptosporidium infection of livestock and poultry in Nanjing Area [49]. Two of 137 fecal samples from Père David's deer have been found to be positive for Cryptosporidium deer genotype in Yancheng with nested PCR [90].

In Zhejiang, varying degrees of infection with Cryptosporidium have been observed in broiler chickens in seven cities at the prefecture level in Zhejiang with the saturated sucrose solution floating method and nested PCR. C. baileyi, C. meleagridis and avian genotype II have been identified [91].

\section{CRYPTOSPORIDIUM CONTAMINATION IN WATER}

The epidemiology of waterborne transmission of Cryptosporidium has been a serious public health concern; thus, many studies have focused on the detection and quantification of Cryptosporidium oocysts in surface, recreational and wastewater matrices through various methodological approaches (concentration, purification and identification), to respond to and prevent potential outbreaks of cryptosporidiosis. Similar surveys have also been performed in the Yangtze River Delta (Fig 5).

In Anhui, only one article has reported investigation of Cryptosporidium contamination in source and tap water in Hefei. The surveys were conducted between August and October 2014 (wet season) and between November and December 2014 (dry season). Cryptosporidium (source water: $26.7 \%$; tap water: $1.7 \%$ ) was detected with the US

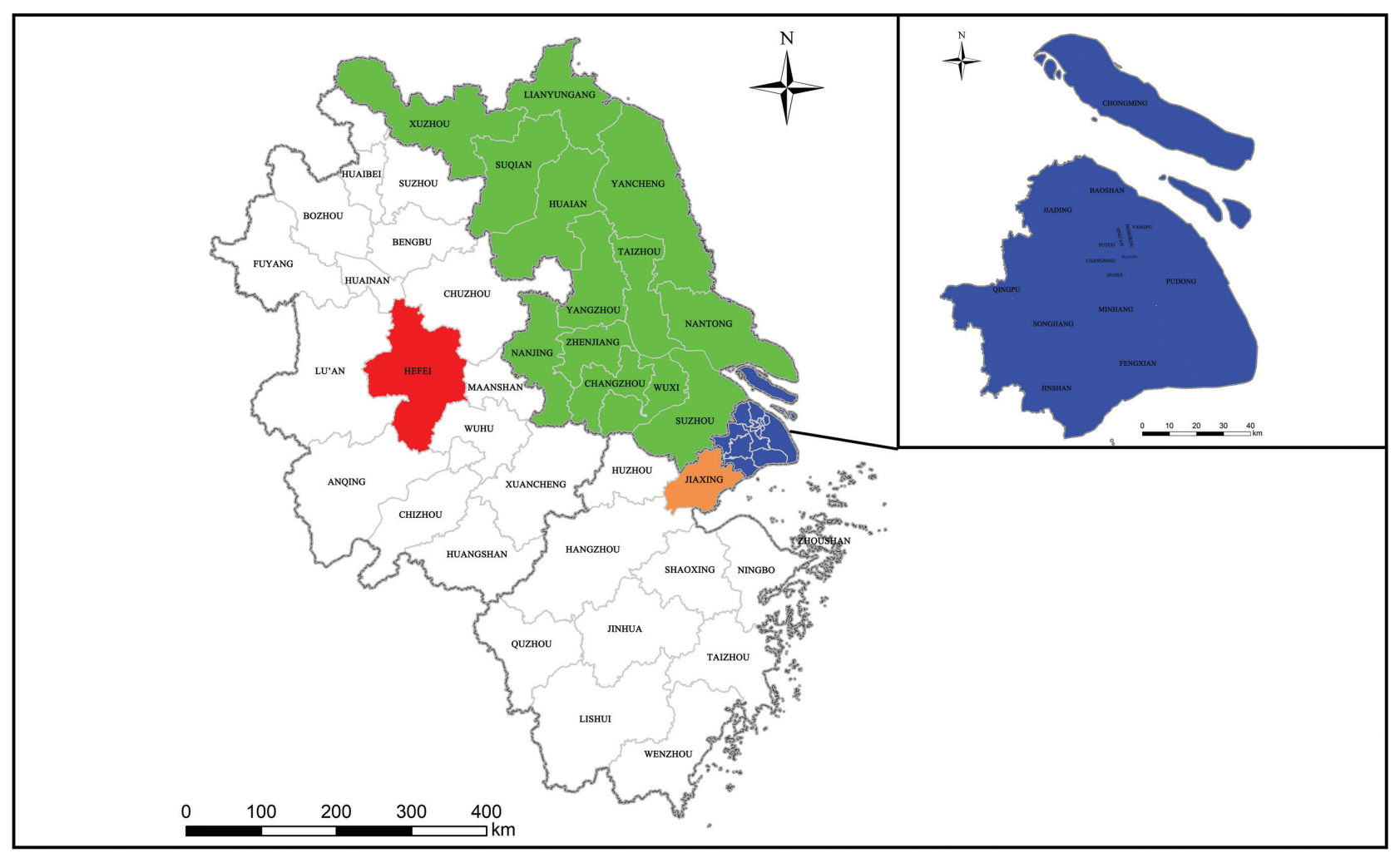

Figure 5 | Cryptosporidium investigations in different types of water at the prefectural level in the Yangtze River Delta since 2001. Red, green, blue and orange represent the prefecture-level cities/districts in Anhui, Jiangsu, Shanghai and Zhejiang, respectively, where the Cryptosporidium surveys in water were performed. 
Environmental Protection Agency (USEPA) Method 1623 during high and low-flow periods [92].

In Jiangsu, drinking source water, treated water and tap water in urban and rural areas have been the focus of monitoring Cryptosporidium contamination, mainly through Method 1623, to assess the potential risk of cryptosporidiosis outbreaks. Two source water samples from Jintan (Changzhou) [93], one source water sample from Nanging, Zhenjiang and Yangzhou each [94], and one treated water from an unreported location [95] have been found to be positive for Cryptosporidium oocysts. Moreover, a 1-year investigation (January to November, 2015) of the occurrence and distribution of Cryptosporidium in Yunlong Lake, Xuzhou, has been performed with Method 1622. In that study, a total of $35 \%$ of water samples $(42 / 120)$ and $46.67 \%$ of sediment samples (28/60) tested positive for Cryptosporidium, and July was associated with the highest risk of both swimming and diving. The concentration of Cryptosporidium oocysts was $0-8 / 10 \mathrm{~L}$ in the water samples and $0-260 / \mathrm{g}$ in sediment samples [96].

In Shanghai, the occurrence of Cryptosporidium in drinking source water, treated water and tap water, and wastewater have been investigated, mainly with Method 1623, and PCR has been used for genotyping and subtyping. Cryptosporidium oocysts have always been detected in the drinking source water (12\%-100\%) [97-102] and wastewater $(14.29 \%-70 \%)$ [67,97,103-106], as compared with treated water and tap water; however, 2 of 30 tap water samples have been found to be positive for Cryptosporidium with Method 1623 in a survey located upstream of Shanghai, at the intake of the drinking water treatment plants in Shanghai [101]. C. andersoni, C. suis, C. baileyi, C. scrofarum, C. meleagridis, C. parvum, C. hominis, C. ryanae, C. fragile, C. cuniculus, rat genotype IV, avian genotype II, avian genotype III, C. hominis IaA18R4, C. parvum IIdA19G1, C. cuniculus VbA20 and C. meleagridis IIIbA24G1 have been identified in drinking source water [101,102], whereas the species C. hominis, C. parvum, C. meleagridis, C. baileyi, C. muris, C. suis, C. viatorum, C. ubiquitum, C. felis, Cryptosporidium $\mathrm{W} 13314$ and genotypes of rat genotype, avian genotype III, rat genotype I, rat genotype IV, a new undetermined genotype and subtypes of C. hominis IaA13R4/ IaA14R 4/IaA18R 4/IaA19R4/IbA19G2/IbA20G2/ IbA21G2/IdA14/IdA16/IdA19/IeA12G3T3/IfA20G1/ IfA22G1, C. parvum IIdA19G1, C. meleagridis IIIbA18G1R1/ IIIbA21G1R1da/IIIbA22G1R1c/IIIbA24G1R1c, C. viatorum XVaA6 and C. ubiquitum XIIg/XIIh have been detected in wastewater [103-105,107].

In Zhejiang, a total of 47 samples collected from three sampling campaigns over the rainy season in 2009 along the Beijing-Hangzhou Grand Canal in Tongxiang have revealed the presence, genotypes and likely sources of Cryptosporidium oocysts in this complicated river network system [108].A total of 37 samples (78.7\%) have been found to be positive, on the basis of immunofluorescence microscopy analyses according to Method 1623, with a mean concentration of 0.51 oocysts per liter. In addition, animal related Cryptosporidium species/genotypes including C. suis, C. fragile and the avian
III, pig II and cervine genotypes have been identified with nested PCR analysis. Three of these species/genotypes have also been detected in wastewater samples from neighboring animal farms, thus indicating that farm animals rather than humans might be the major pollution sources.

\section{CONCLUSIONS}

Cryptosporidium is widespread in humans, animals and water bodies in the Yangtze River Delta, and most of the zoonotic species (C. hominis, C. parvum, C. meleagridis, C. andersoni, C. muris, C. suis, C. viatorum, C. ubiquitum, C. felis, C. scrofarum, C. xiaoi, C. bovis, C. cuniculus and C. canis) reported in this region pose a major public health threat, given that most have been identified in water. Moreover, because Cryptosporidium species and genotypes are more abundant in water than in human and animal sources, many potential sources of infection have not been identified. The following issues are worthy of attention in this region: (1) Although numerous surveys on Cryptosporidium infection have been conducted, no region-wide study on the prevalence of Cryptosporidium infection has been performed. (2) Most reports have been from scattered cross-sectional surveys performed on several hosts or water bodies in different areas, but no systematic surveillance has been conducted at the human-animalenvironment interface, even for only humans, makes it difficult to explain the route of transmission. (3) Despite the extensive and increasing prevalence of cryptosporidiosis, many deficiencies exist in the available diagnostic tools. Microscopic techniques remain the most widely used method for the detection of Cryptosporidium oocysts in fecal samples, particularly those from humans, but misdiagnosis of the disease is common, because the diagnostic accuracy is largely dependent on experience. (4) Cooperative work on the surveillance of cryptosporidiosis within and between provinces is lacking. Agreement is far from being reached regarding the establishment of an integrated health service system with a unified public network for infectious disease prevention and control in the Yangtze River Delta. (5) The One Health approach has not been fully used to address Cryptosporidium infection (or cryptosporidiosis), and no data on other transmission factors beyond humans, animals and water have been reported.

Therefore, advancing Cryptosporidium molecular detection technology is necessary, as is improving research and development for new detection technology. Meanwhile, a comprehensive infectious disease surveillance network including cryptosporidiosis and other neglected tropical diseases should be established to achieve real-time detection and monitoring according to the One Health approach at the human-animal-environment-food-others interface in the Yangtze River Delta. In addition, increasing multisectoral cooperation, and exchanging and sharing information within and between provinces, is also necessary. Together, these efforts should greatly contribute to the integration of the control and prevention of infectious diseases with high-quality development, to benefit millions of residents. 


\section{ACKNOWLEDGEMENTS}

This work was supported by the National Science and Technology Major Project, China (No. 2018ZX10201002-009 to JY) and the Three-Year Public Health Action Plan of Shanghai (2020-2022) (No. GWV-10.1-XK13 to JC). The funders had no role in the study design, data collection and analysis, decision to publish or preparation of the manuscript.

\section{CONFLICTS OF INTEREST}

The authors declare no competing interests.

\section{REFERENCES}

1. Ryan UM, Feng Y, Fayer R, Xiao L. Taxonomy and molecular epidemiology of Cryptosporidium and Giardia - a 50 year perspective (1971-2021). Int J Parasitol. 2021;51:1099-1119.

2. Pumipuntu N, Piratae S. Cryptosporidiosis: a zoonotic disease concern. Vet World. 2018;11(5):681-686.

3. Ryan U, Zahedi A, Feng Y, Xiao L. An update on zoonotic Cryptosporidium species and genotypes in humans. Animals. 2021;11(11):3307

4. Feng Y, Ryan UM, Xiao L. Genetic diversity and population structure of Cryptosporidium. Trends Parasitol. 2018;34(11):997-1011.

5. Savioli L, Smith H, Thompson A. Giardia and Cryptosporidium join the 'Neglected Diseases Initiative'. Trends Parasitol. 2006;22(5):203-208.

6. Collaborators GBDDD. Estimates of global, regional, and national morbidity, mortality, and aetiologies of diarrhoeal diseases: a systematic analysis for the Global Burden of Disease Study 2015. Lancet Infect Dis. 2017;17(9):909-948.

7. Checkley W, White AC Jr, Jaganath D, Arrowood MJ, Chalmers RM, Chen XM, et al. A review of the global burden, novel diagnostics, therapeutics, and vaccine targets for Cryptosporidium. Lancet Infect Dis. 2015;15(1):85-94.

8. O'Leary JK, Sleator RD, Lucey B. Cryptosporidium spp. diagnosis and research in the 21st century. Food Waterborne Parasitol. 2021;24:e00131.

9. Fayer R. Cryptosporidium: a water-borne zoonotic parasite. Vet Parasitol. 2004;126(1-2):37-56.

10. Zahedi A, Ryan U. Cryptosporidium - an update with an emphasis on foodborne and waterborne transmission. Res Vet Sci. 2020;132:500-512.

11. Hassan EM, Ormeci B, DeRosa MC, Dixon BR, Sattar SA, Iqbal A. A review of Cryptosporidium spp. and their detection in water. Water Sci Technol. 2021;83(1):1-25.

12. Ryan U, Zahedi A, Paparini A. Cryptosporidium in humans and animals - a One Health approach to prophylaxis. Parasite Immunol. 2016;38(9):535-547.

13. Innes EA, Chalmers RM, Wells B, Pawlowic MC. A One Health approach to tackle cryptosporidiosis. Trends Parasitol. 2020;36(3):290-303.

14. Wang Y, Zhang K, Chen Y, Li X, Zhang L. Cryptosporidium and cryptosporidiosis in wild birds: a One Health perspective. Parasitol Res. 2021;120(9):3035-3044.

15. Blake DP, Betson M. One Health: parasites and beyond. Parasitology. 2017;144(1):1-6.

16. Liu A, Gong B, Liu X, Shen Y, Wu Y, Zhang W, et al. A retrospective epidemiological analysis of human Cryptosporidium infection in China during the past three decades (1987-2018). PLoS Negl Trop Dis. 2020;14(3):e0008146.

17. Han F, Tan W, Zhou X. [Two case reports of cryptosporidiosis in Nanjing]. Jiangsu Med J. 1987;12:692.

18. Xie SY. [Analysis of opportunistic infection in 139 patients with AIDS]. J Qiqihar Univ Med. 2011;32(13):2080-2081.

19. Tian LG, Chen JX, Wang TP, Cheng GJ, Steinmann P, Wang FF, et al. Co-infection of HIV and intestinal parasites in rural area of China. Parasit Vectors. 2012;5:36.
20. Tian LG, Cheng GJ, Chen JX, Cai YC, Guo J, Tong XM, et al. [Survey on co-infection with HIV and intestinal parasites in high prevalence areas of HIV/AIDS, China]. Zhongguo Xue Xi Chong Bing Fang Zhi Za Zhi. 2012;24(2):168-172.

21. Tian LG, Wang TP, Cheng GJ, Wang FF, Tong XM, Guo $J$, et al. [Cross-section study on co-infection of HIV and Cryptosporidium]. Zhongguo Xue Xi Chong Bing Fang Zhi Za Zhi. 2012;24(1):54-57, 61

22. Tian LG, Wang TP, Lv S, Wang FF, Guo J, Yin XM, et al. HIV and intestinal parasite co-infections among a Chinese population: an immunological profile. Infect Dis Poverty. 2013;2(1):18.

23. Xu LF, Wang J, Wang KX, Li CP. [Cryptosporidium infection among students in Anhui Province]. Chin J School Health. 2003;24(4):347-348.

24. Xu LF, Lu J, Li CP. [Epidemiological survey of Cryptosporidium infection among children in 29 kindergartens in Anhui Province]. Chin J Epidemiol. 2004;25(4):336.

25. Lu J, Li CP, Yang ZH, Zhang LH. An epidemiological survey of cryptosporidiosis in Huainan District. J Huainan Inst Technol. 2001;21(4):71-72.

26. Cai R, Li CP, Wang J, Xu LF, He J. [An epidemiological survey of cryptosporidiosis with diarrhea in Huainan area]. J Trop Dis Parasitol. 2003; 1(1):26-28.

27. Wang QQ, Guo JD, Cao ZG, Wang QZ, Liu DH, Wang TP. [Investigation on human Cryptosporidium infection in local area of Anhui Province]. Zhongguo Xue Xi Chong Bing Fang Zhi Za Zhi. 2015;27(3):263-267, 272.

28. Zhan W, Chen YG, Jing LY, He SH, Liang ZH. [Investigation of Cryptosporidium infection rate among patients with diarrhea in four cities in China]. Chin J Control Prev. 2002;6(1):76-77.

29. Jiang Y, Ren J, Yuan Z, Liu A, Zhao H, Liu H, et al. Cryptosporidium andersoni as a novel predominant Cryptosporidium species in outpatients with diarrhea in Jiangsu Province, China. BMC Infect Dis. 2014;14:555.

30. Du XL, Ge JJ, Qin YF, Meng R, Liu Y, Chu K, et al. [The epidemiological study on cryptosporidiosis in outpatients of Nanjing Children's Hospital]. J Trop Med. 2009;9(4):382-286.

31. Wang L, Tian XH, He ZA, He CY, Ding Z, Xia YT, et al. [Cryptosporidium infection of children in some rural areas of China]. J Environ Hyg. 2015;5(4):328-331.

32. Zheng H, Wang L, Zhang R, Ding Z. [Cryptosporidium contamination in rural water supplies and Cryptosporidium infestation among children in Jiangsu Province]. J Environ Hyg. 2015;5(5):418-421.

33. Yang PC, Zhang HY, Zhou W, Yin WG, Wu P, Zhang K, et al. [Cryptosporidium infection in Nanjing City from 2015 to 2016]. Zhongguo Xue Xi Chong Bing Fang Zhi Za Zhi. 2017;29(6):752-755.

34. Zheng H, He J, Wang L, Zhang R, Ding Z, Hu W. Risk factors and spatial clusters of Cryptosporidium infection among school-age children in a rural region of eastern China. Int J Environ Res Public Health. 2018;15(5).

35. Zhu HS, Du XL, Yu RB, Xu JM, Zhu LF, Wu HW. [Serum immunology investigation of Cryptosporidium infection among injection drug abusers]. Zhongguo xue xi chong bing fang zhi za zhi. 2008;20(5):364-366.

36. Zhou HF, Zhu M, Yuan JL, Xu F, Chen YH, Zhang ZX, et al. [A survey on Cryptosporidium parvum cryptosporidiosis in different people of Luwan district Shanghai]. Sh J Prev Med. 2005;17(9):430-432.

37. Qian FX, Shi M, Shen SL, Liu YH, Gao T. [Investigation of Cryptosporidium infection in adults with non-AIDS and AIDS diarrhea]. Chin J Misdiagn. 2011;11(3):752.

38. Zhang $X P$, He $Y Y$, Wang ZY, Zhang YG, Zhu Q, Jiang SF, et al. [Investigation on Cryptosporidium infections in patients with diarrhea from the general hospitals in Shanghai]. China Trop Med. 2016;16(2):1183-1186.

39. Liu $H$, Shen $Y$, Yin J, Yuan $Z$, Jiang $Y, X u Y$, et al. Prevalence and genetic characterization of Cryptosporidium, Enterocytozoon, 
Giardia and Cyclospora in diarrheal outpatients in China. BMC Infect Dis. 2014;14:25.

40. Feng $Y$, Wang L, Duan L, Gomez-Puerta LA, Zhang L, Zhao $X$, et al. Extended outbreak of cryptosporidiosis in a pediatric hospital, China. Emerg Infect Dis. 2012;18(2):312-314.

41. Li C, Li XY, Fang ZX. [A case of intracranial infection with Cryptosporidium]. Chin J Zoonoses. 2003;19(1):131-133.

42. Cai Y, Zhang NZ, Gong QL, Zhao Q, Zhang XX. Prevalence of Cryptosporidium in dairy cattle in China during 2008-2018: a systematic review and meta-analysis. Microb Pathog. 2019;132:193-200.

43. Gong C, Cao XF, Deng L, Li W, Huang XM, Lan JC, et al. Epidemiology of Cryptosporidium infection in cattle in China: a review. Parasite. 2017;24:1.

44. Sun T, Liu W, Wang JH, Xue XH, Zhao CC, Li PY. [Isolation and identification of cow-origin Cryptosporidium isolates in Hefei]. Zhongguo Ji Sheng Chong Xue Yu Ji Sheng Chong Bing Za Zhi. 2011;29(6):447-452.

45. Wu L, Li PY, Liu QS, Xu QM, Yu WY. [Epidemiological study of cattle cryptosporidiosis in a Hefei dairy farm]. China Dairy Cattle. 2006;(4):40-41.

46. Zhou QX, Li JC, Li PY, Xu QM, Yu LP, Liu QS, et al. [Epidemiological investigation of cow cryptosporidiosis at a cow dairy farm in Hefei]. J Hebei Agri Sci. 2008;12(1):122-124.

47. Xu WL, Li PY, Gu YF, Xu QM, Zhao CC, Liu QS, et al. [Epidemiological survey of cow cryptosporidiosis in Anhui Province]. J Anhui Sci Technol Univ. 2007;21(6):9-11.

48. Li PY, Lu FL, Li JN, Jiang H, Wang M, Liu GL. [Epidemiological investigation of cryptosporidiosis in cattle in Anhui Province]. Anim Husb Vet Med. 2003;35(3):13-15.

49. Chen F, Huang KH. [Investigation on Cryptosporidium infection of livestock and poultry in Nanjing area]. Anim Husb Vet Med. 2012;44(12):67-69.

50. Zhu D, Lv YL, Li MJ, Zhang ZJ, Jian FC, Song D, et al. [Survey on the prevalence of intestinal parasites in goats in partial regions of China]. China Herbivore Sci. 2013;33(1):43-46.

51. Zhang HY, Wang XT, Niu WJ, WEI DH, YANG PC, Zong ZM, et al. [Etiology of Cryptosporidium infection in a dairy farm in Nanjing]. Anim Husb Vet Med. 2013;45(12):98-99.

52. Liu YX, Cui HP, Zhou YH, Fang F. [Preliminary investigation on Cryptosporidium infection of milch COWS in Wuxi areas]. Zhongguo Xue Xi Chong Bing Fang Zhi Za Zhi 2005;17(3).

53. Li N, Wang R, Cai M, Jiang W, Feng Y, Xiao L. Outbreak of cryptosporidiosis due to Cryptosporidium parvum subtype IIdA19G1 in neonatal calves on a dairy farm in China. Int J Parasitol. 2019;49(7):569-577.

54. Xu MQ, Zhu SH, Huang Y, Huang X, Wang ZJ, Gao XC, et al. [Investigation on current status of Cryptosporidium infection in dairy cows in Shanghai]. Sh Anim Husb Vet News. 2007(3):36

55. Cai M, Guo Y, Pan B, Li N, Wang X, Tang C, et al. Longitudinal monitoring of Cryptosporidium species in pre-weaned dairy calves on five farms in Shanghai, China. Vet Parasitol. 2017:241:14-19.

56. Chen F, Huang K. Prevalence and molecular characterization of Cryptosporidium spp. in dairy cattle from farms in China. J Vet Sci. 2012;13(1):15-22.

57. Yang XY, Gong QL, Zhao B, Cai YN, Zhao Q. Prevalence of Cryptosporidium infection in sheep and goat flocks in China during 2010-2019: a systematic review and meta-analysis. Vector Borne Zoonotic Dis. 2021;21(9):692-706.

58. Mi R, Wang $X$, Huang $Y$, Mu G, Zhang $Y$, Jia $H$, et al. Sheep as a potential source of zoonotic cryptosporidiosis in China. Appl Environ Microbiol. 2018;84(18).

59. Li WC, Wang K, Tang L, Chen M, Li H, Kan ZZ, et al. [Molecular characterization of Cryptosporidium species in sheep and goats in Anhui Province and neighboring provinces]. Zhongguo Xue Xi Chong Bing Fang Zhi Za Zhi. 2019;31(5):474-478.
60. Gu YF, Li WC, Wang LK, Li Y, Li L, Chu XH, et al. [Investigation on intestinal parasite infection of goats in Anhui Province]. Anim Husb Vet Med. 2014;46(10):100-103.

61. Mi R, Wang X, Huang Y, Zhou P, Liu Y, Chen Y, et al. Prevalence and molecular characterization of Cryptosporidium in goats across four provincial level areas in China. PLoS One. 2014;9(10):e111164.

62. Wang W, Gong QL, Zeng A, Li MH, Zhao Q, Ni HB. Prevalence of Cryptosporidium in pigs in China: a systematic review and meta-analysis. Transbound Emerg Dis. 2021;68(3):1400-1413.

63. Zhao CC, Li PY, Wu L, Chen LZ, Yu WY. [Epidemiology survey on swine cryptosporidiosis in Anhui province]. Anim Husb Vet Med. 2005;37(10):18-20

64. Li WC, Yang HH, Kan ZZ, Yang YL, Sun YY, Gu YF, et al. [Prevalence of Cryptosporidium spp. infections in large-scale pig farms in north of Yangtze River in Anhui Province]. Zhongguo Xue Xi Chong Bing Fang Zhi Za Zhi. 2018;30(4):420-423.

65. Zhao CC, Li PY. [Epidemiological investigation of swine cryptosporidiosis in Fengtai County, Anhui Province]. Chin J Vet Parasitol. 2003;11(1):42-44.

66. Chen F, Huang K. Prevalence and phylogenetic analysis of Cryptosporidium in pigs in eastern China. Zoonoses Public Health. 2007;54(9-10):393-400.

67. Shen LP, Liu PH, Xue X, Chen ZG, Mi RS. [Investigation on the infection status of swine Cryptosporidium in Shanghai]. Sh Anim Husb Vet News. 2009;(2):32-33.

68. Chen Z, Mi R, Yu H, Shi Y, Huang Y, Chen Y, et al. Prevalence of Cryptosporidium spp. in pigs in Shanghai, China. Vet Parasitol. 2011;181(2-4):113-119.

69. Yin J, Shen Y, Yuan Z, Lu W, Xu Y, Cao J. Prevalence of the Cryptosporidium pig genotype II in pigs from the Yangtze River Delta, China. PLoS One. 2011;6(6):e20738.

70. Yin JH, Yuan ZY, Cai HX, Shen YJ, Jiang YY, Zhang J, et al. Agerelated infection with Cryptosporidium species and genotype in pigs in China. Biomed Environ Sci. 2013;26(6):492-495.

71. Liu H, Ni H, Xu J, Wang R, Li Y, Shen Y, et al. Genotyping and zoonotic potential of Cryptosporidium and Enterocytozoon bieneusi in pigs transported across regions in China. Microb Pathog. 2021;154:104823.

72. Wang XL, Li PY, Gu YF, Xu QM, Zhao CC, Liu W. [Investigation into Cryptosporidium infection on primates and herbivores in a zoo in Anhui]. J Anhui Sci Technol Univ. 2010:24(1):8-11.

73. Wang GH, Zhang ZZ. [Investigation on the prevalence of Cryptosporidium infection in primates in Hefei Wildlife Park]. Anim Husb Feed Sci. 2018;39(7):109-112.

74. Wang GH. [Investigation on intestinal parasite infection of some animals in Hefei Wildlife Park]. Shou Yi Dao Kan. 2021;(2):126-127,143.

75. Gu Y, Wang X, Zhou C, Li P, Xu Q, Zhao C, et al. Investigation on Cryptosporidium infections in wild animals in a zoo in Anhui Province. J Zoo Wildl Med. 2016;47(3):846-854.

76. Yu LP, Li PY, Gu YF, Xu WL, Wang XL, Qiu XB, et al. [Epidemiological survey dog cryptosporidiosis in Hefei]. J Anhui Sci Technol Univ. 2009;23(1):15-18.

77. Wang JH, Li PY, Xue XH, Chen XM, Jiang SD, Qiu XB, et al. [Investigation on the infection situation of Cryptosporidium in dogs in Hefei city]. Chin J Vet Parasitol. 2008;16(5):20-23.

78. Gu YF, Wang K, Liu DY, Mei N, Chen C, Chen T, et al. [Molecular detection of Giardia lamblia and Cryptosporidium species in pet dogs]. Chin J Parasitol Parasit Dis. 2015;33(5):362-367.

79. Li W, Liu X, Gu Y, Liu J, Luo J. Prevalence of Cryptosporidium, Giardia, Blastocystis, and trichomonads in domestic cats in East China. J Vet Med Sci. 2019;81(6):890-896.

80. Dang HL, He GS, Zhang LX, Cao J, Jin HY, Yu JY, et al. [Investigation on the prevalence of Cryptosporidium in reptiles and amphibians in Shanghai]. Chin J Zoonoses. 2008;24(2):179-181 
81. Zhang SR, Shen YJ, Wang B, Liu H, Cao SK, Yuan ZY, et al. [Investigation of Cryptosporidium infection in primates in a zoo of Shanghai]. Chin J Parasitol Parasit Dis. 2017;35(2):136-139.

82. Yin J, Yuan Z, Shen Y, Zhang J, Jiang Y, Cao J. Molecular identification of Cryptosporidium spp. from animal sources in China. J Infect Dev Ctries. 2013;7(12):1020-1022.

83. Zhang Y, Mi R, Yang L, Gong H, Xu C, Feng Y, et al. Wildlife is a potential source of human infections of Enterocytozoon bieneusi and Giardia duodenalis in southeastern China. Front Microbiol. 2021;12:692837.

84. Shen LP, Wang XX, Xu F, Mi RS, Gong GH, Xia LM, et al. [Investigation on Cryptosporidium infection in dog reclamation bases in Shanghai]. Chin J Animal Husb Vet Med. 2020;(12):46-48.

85. Han HX, Cheng Q, Sun $A B$, Xing $T$, Chen MS, Sun Q, et al. [Epidemiological survey of canine cryptosporidiosis in Minhang District, Shanghai]. Sh Anim Husb Vet News. 2021;(3):45-48.

86. Xu H, Jin Y, Wu W, Li P, Wang L, Li N, et al. Genotypes of Cryptosporidium spp., Enterocytozoon bieneusi and Giardia duodenalis in dogs and cats in Shanghai, China. Parasit Vectors. 2016;9:121.

87. Karim MR, Zhang S, Jian F, Li J, Zhou C, Zhang L, et al. Multilocus typing of Cryptosporidium spp. and Giardia duodenalis from non-human primates in China. Int J Parasitol. 2014;44(13):1039-1047.

88. Wei JZ, Li PY, He CS, Zhu QY, Xie JG. [Investigation on infectious condition of chicken Cryptosporidium in a private farm of Anhui Province]. Chin J Vet Sci. 2003;23(5):472-473.

89. Huang JM, Chen HL, Zhou YK, Wang S, Ren Q, Fang Z, et al. The first report of Cryptosporidium testudinis in Chinese alligators (Alligator sinensis) in China. Parasitol Res. 2020;119(7):2359-2362

90. Huang SY, Fan YM, Yang Y, Ren YJ, Gong JZ, Yao N, et al. Prevalence and molecular characterization of Cryptosporidium spp. in Pere David's deer (Elaphurus davidianus) in Jiangsu, China. Rev Bras Parasitol Vet. 2020;29(2):e017919.

91. Wang L, Xue X, Li J, Zhou Q, Yu Y, Du A. Cryptosporidiosis in broiler chickens in Zhejiang Province, China: molecular characterization of oocysts detected in fecal samples. Parasite. 2014;21:36

92. Zhang $X$, Wu LP, Lv JT, Wang ZQ, Fu HY, Sun ZK, et al. [Investigation on contamination of Cryptosporidium and Giardia in source and pipe water from some cities of China]. J Environ Health. 2016;33(1):41-43.

93. Cao SK, Jiang YY, Yuan ZY, Yin JH, Xu M, Xue JB, et al. Quantitative microbial risk assessment of Cryptosporidium and Giardia in public drinking water in China. Biomed Environ Sci. 2021;34(6):493-498.

94. Ni BX, Shen $M X, X u X Z$, Wang $X T$, Dai $Y$, Jin XL. [Investigation on contamination of Giardia and Cryptosporidium in drinking water in Jiangsu Province]. Zhongguo Xue Xi Chong Bing Fang Zhi Za Zhi. 2017;29(4):454-458.
95. Zheng $\mathrm{H}$, Yu Y, Fei J, Wang L, Zhang R, Ding Z, et al. [Investigation on Cryptosporidium and Giardia contamination in source water and drinking water in Jiangsu Province]. J Environ Hyg. 2014;4(6):535-537.

96. Kong Y, Lu P, Yuan T, Niu J, Li Z, Yang B. Cryptosporidium contamination and attributed risks in Yunlong Lake in Xuzhou, China. Can J Infect Dis Med Microbiol. 2017;2017:4819594.

97. Bai XH, Zeng L, Zhu B, Wang HL. [Existence of Cryptosporidium and Giardia in the effluent from a WWTP and its receiving water in Shanghai]. Chin J of Health Lab Technol. 2006;16(1):4-5.

98. Zhou Y, Rong Y, Sun L, Lu J, HE Y, Li C, et al. [Investigation on removal of Giardia and Cryptosporidium in Pudong Waterworks in Shanghai]. Water Wastewater Eng. 2007;33(8):16-18.

99. Meng MQ, Jiang ZH, Chen GG. [Investigation on distribution of Cryptosporidium and Giardia in tap \& raw water in Shanghai proper]. China Water Wastewater. 2005;21(12).

100. Zhang XP, He YY, Zhu Q, Ma XJ, Cai L. [Investigation on contamination of Cryptosporidium and Giardia in drinking water and environmental water in Shanghai]. Chin J Parasitol Parasit Dis. 2010;28(6):435-438.

101. Feng Y, Zhao X, Chen J, Jin W, Zhou X, Li N, et al. Occurrence, source, and human infection potential of Cryptosporidium and Giardia spp. in source and tap water in Shanghai, China. Appl Environ Microbiol. 2011;77(11):3609-3616.

102. Hu Y, Feng $Y$, Huang $C$, Xiao L. Occurrence, source, and human infection potential of Cryptosporidium and Enterocytozoon bieneusi in drinking source water in Shanghai, China, during a pig carcass disposal incident. Environ Sci Technol. 2014;48(24):14219-14227.

103. Jiang W, Roellig DM, Li N, Wang L, Guo Y, Feng Y, et al. Contribution of hospitals to the occurrence of enteric protists in urban wastewater. Parasitol Res. 2020;119(9):3033-3040.

104. Li N, Xiao L, Wang L, Zhao S, Zhao X, Duan L, et al. Molecular surveillance of Cryptosporidium spp., Giardia duodenalis, and Enterocytozoon bieneusi by genotyping and subtyping parasites in wastewater. PLoS Negl Trop Dis. 2012;6(9):e1809.

105. Huang C, Hu Y, Wang L, Wang Y, Li N, Guo Y, et al. Environmental transport of emerging human-pathogenic Cryptosporidium species and subtypes through combined sewer overflow and wastewater. Appl Environ Microbiol. 2017;83(16).

106. Ma J, Feng Y, Hu Y, Villegas EN, Xiao L. Human infective potential of Cryptosporidium spp., Giardia duodenalis and Enterocytozoon bieneusi in urban wastewater treatment plant effluents. J Water Health. 2016;14(3):411-423.

107. Feng Y, Li N, Duan L, Xiao L. Cryptosporidium genotype and subtype distribution in raw wastewater in Shanghai, China: evidence for possible unique Cryptosporidium hominis transmission. J Clin Microbiol. 2009;47(1):153-157.

108. Xiao S, An W, Chen Z, Zhang D, Yu J, Yang M. Occurrences and genotypes of Cryptosporidium oocysts in river network of southern-eastern China. Parasitol Res. 2012;110(5):1701-1709.

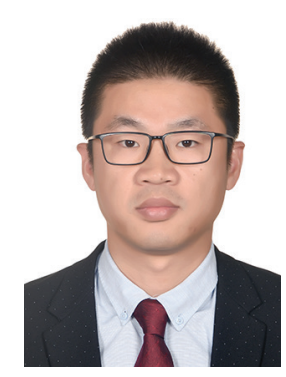

Dr. Jianhai Yin obtained his PhD in 2017, at the National Institute of Parasitic Diseases (NIPD), Chinese Center for Disease Control and Prevention. He has been studying molecular epidemiology, molecular diagnostics, and host-parasite interaction of important and emerging parasitic diseases such as malaria, echinococcosis and cryptosporidiosis since he joined NIPD in 2011. 